\title{
Examination of the Computed Molecular Properties of Compounds Selected for Clinical Development
}

\author{
James F. Blake \\ Array BioPharma, Boulder, CO, USA
}

BioTechniques 34:S16-S20 (June 2003)

\begin{abstract}
We have conducted a systematic evaluation of the calculated molecular properties of compounds in clinical development and have found that the development process selects for compounds that have certain computed physical properties. In particular, as the stage of development progresses, compounds that are advanced have lower calculated octanol/water partition coefficient (ClogP), polar surface area, and molecular weight. The findings of this study provide guidance for combinatorial library design and lead selection, which may enhance the chance for ultimate success in lead optimization.
\end{abstract}

\section{INTRODUCTION}

In the late 1980s, the advent of random high-throughput screening (HTS) as a means of finding novel lead compounds introduced substantial changes in the way pharmaceutical companies view their compound collections (1). Since most in-house collections came primarily from either traditional synthetic techniques or targeted acquisitions, the size of these collections tended to be rather modest by today's standards. The success of random HTS at finding novel leads relies on assaying much larger numbers of compounds, typically in the hundreds of thousands (2). From an analysis of 56 high-throughput screens carried out on a variety of enzymes, $G$ protein-coupled receptors, ion channels, and receptor targets, Spencer concluded that an average of one quality lead series is identified for every 120,000 compounds assayed (3). The link between deriving leads from HTS and the size of the compound collection screened led to a substantial increase in the number of compounds purchased from outside sources. Lipinski has suggested that this increased reliance on leads derived from HTS also led to the pursuit of compounds with less than optimal physicochemical properties and, hence, to more difficulty in lead optimization and clinical development (4). Experience has shown that improving the oral bioavailability of a compound that has already been optimized for potency is the most time-consuming, labor-intensive, and variable part of the preclinical drug discovery process (5).

These observations led to the creation of the "rule-of-five" by Lipinski et al. (6). Lipinski and coworkers compared drug-like compounds (those with U.S. Adopted Names [USAN] or International Nonproprietary Names [INN] names extracted from the World Drug Index) with compounds not presumed to be drug-like (those found in the Available Chemical Directory [ACD]) and determined that compounds with excessive $\log P$, molecular weight (MW), hydrogen-bond donors, or acceptors were more likely to have solubility and/or permeability problems that would lead to poor oral bioavailability (6). Since the publication of the rule-of-five, there have been many additional studies aimed at distinguishing between drugs and nondrugs (7-9), characterizations of the properties of drug-like databases (10-13), and the prediction of oral bioavailability (14-16). From these exercises, one would hope to define the appropriate molecular properties from which leads or compounds targeted for clinical investigation should be derived.

A different perspective on appropriate characteristics of leadlike compounds was obtained by examining the property distributions of drugs and the corresponding lead structures. Studies by Oprea et al. (17) and Hann et al. (18) demonstrated that the process of optimizing leads into drugs can result in noticeable increases in $\mathrm{MW}, \log P$, and other important physicochemical parameters. A similar analysis of drugs launched in 2000 and the corresponding lead structures by Proudfoot (19) found that leads are very similar to the final drugs both in terms of structure and simple physical properties. For the 29 series of compounds in that study, the leads and drugs were generally within $25 \%$ of the MW and one calculated $\log P$ unit of each other. Many of the "leads" in this study were actually marketed drugs. This result is consistent with the earlier analyses $(17,18)$ and underscores the importance of starting with the best lead compounds. The implicit belief is that higher quality leads should ultimately give rise to clinical candidates with higher success rates and better survivability prospects.

The studies cited above have provided the groundwork for classifying leads and drugs and have determined the parameters that are important for describing drug-like compounds. The current study asks the question "Does clinical development select for compounds with certain physical properties?" We will answer this question by comparing the computed properties of compounds at various stages of clinical development. 
Table 1. Classes of Compounds Eliminated from this Study

\begin{tabular}{|c|c|}
\hline Anesthetic & -Magnetic resonance imaging agent \\
\hline .Antibacterial (ophthalmic) & -Mediator release inhibitor \\
\hline Glycopeptide & -Metabolism regulator \\
\hline -Antidote (specific) & -Metal complex \\
\hline -Antihematotoxic agent & -Mineral \\
\hline -Antioxidant & -Nondepolarizing blocking drug \\
\hline Blood substitute & .Pharmaceutical aid \\
\hline Peptide agonists and antagonists & Photosensitizer \\
\hline Chemopreventive & Platinum complex \\
\hline Chemoprotective & Pulmonary surfactant \\
\hline Chemical delivery system & -Radiopharmaceuticals \\
\hline Choleretic & Radioprotector \\
\hline Chromium complex & -Radiosensitizer \\
\hline Contrast medium & -Retinoprotector \\
\hline Corneal wound healing stimulator & Spermicide \\
\hline Diagnostic agents & Sweetener \\
\hline Disease modifying drug & Ultraviolet light absorber \\
\hline .Drug delivery system & Uterine stimulant \\
\hline Expectorant & Vaccine \\
\hline Isotope & -Vitamins \\
\hline $\begin{array}{l}\text {-Kidney function improver } \\
\text { Liposome composition }\end{array}$ & .Wound healing agent \\
\hline
\end{tabular}

\section{METHODS}

For our study, the drug and drug-like compounds are derived from the MDL Drug Data Report (MDDR)-99.2 database (20). The MDDR database contains compounds that were targeted and tested for potential therapeutic value and were compiled from various journals, meetings, and patent literature. The compounds are also categorized according to the stage of clinical or biological investigation. Given the extremely complex nature of drug development, this database represents a "snapshot" of compounds at various stages. The value lies in finding trends in the data. The MDDR database contains a total of 105,084 compounds. Of these compounds, 95,151 fall into the class of "biological testing". For our purposes, we are only interested in compounds that have been chosen for clinical investigation; these classes include Preclinical, Phase I, Phase II, Phase III, and Launched. We eliminated all compounds for which no therapeutic activity class was given, as well as compounds that fall into certain classes (see Table 1). These exclusions were meant to remove diagnostics, topical agents, and compounds not targeted toward a specific disease state. After this process, the MDDR clinical database contained 6467 entries that are biased toward orally available agents.

For each of the compounds in the data set, we computed a number of properties that have been shown to be important for characterizing leads and drug-like molecules, such as polar surface area (PSA) (21-23). Andrews' binding energy (24), rotatable bond counts (16), calculated octanol/water partition coefficient $(C \log P)$, and MW (6). For each structural entry in the database, all counter-ions (salts, hydrates, etc.) were removed, and the remaining single molecule was converted to the neutral form. The relevance of these properties to the processes of solubility, permeability, and absorption has been recently reviewed $(8,21-23)$. These properties are also the most amenable to change via synthetic design and are potentially "fixable" in the lead optimization process.

The 3-dimensional structure of each compound was computed with the CONCORD program (CONCORD, version 4.06; Tripos, St. Louis, MO, USA). The PSA was computed from the solvent accessible surface (probe radius of $1.4 \AA$ ) with an in-house program that utilizes the SAVOL2 algorithm (25). Polar atoms contributing to the PSA include N, $\mathrm{O}$, attached hydrogens, and carbons involved in carbonyl or imide moieties. The $\log P$ was computed with the $\mathrm{Clog} P$ program $(\mathrm{C} \log P$, version 4.0 [version 18 fragment database]; Tripos). All additional parameters were computed via Sybyl with appropriate Sybyl Programming Language (SPL) scripts (Sybyl, version 6.8; Tripos). All statistical analysis was carried out with the program $R(26)$.

\section{RESULTS}

The results of the calculations on the 6474 compounds are summarized according to the property and phase of clinical investigation in Table 2. Both Shapiro-Wilk tests (27) and quartile-quartile normality plots revealed that the values of computed properties in each group are not normally distributed, thus median values for each property should be used for comparison purposes, since mean values will tend to be overly influenced by the skewed distributions. Also included in Table 2 is the percentage change that is observed in each property on advancing from Preclinical to Launched status (\% Change). While median values for a property are useful, it is also important to consider how compounds fare when they possess out-of-range values. For a rule-of-five analysis, these would include both $\operatorname{Clog} P>5$ and $\mathrm{MW}>500$. Other studies have shown that increasing the PSA beyond $150-200 \AA^{2}(2,5,22)$ or rotatable bonds $>10$ (16) can lead to decreased permeability and oral bioavailability. The percentage of compounds that fall outside each of these cutoff values is also reported in Table 2. From inspection of Table 2 for each of the properties listed, there is a noticeable decrease in the median value of each property as compounds advance from Preclinical to Launched status. To judge the possible significance of these changes, a pair-wise Wilcoxon-Mann-Whitney test was performed on each computed property group with a standard $95 \%$ confidence level and pooled $P$ values (28). The pair-wise Wilcoxon-Mann-Whitney test for significance was used since no assumptions are made regarding the normality of the distribution nor its variance. Each group consisted of the individual computed properties, partitioned according to the phase of development. These tests revealed that the differences observed for the median values listed 
Table 2. Calculated Molecular Properties of Compounds Under Clinical Investigation

\begin{tabular}{|c|c|c|c|c|c|c|}
\hline Propertya & Preclinical & Phase I & Phase II & Phase III & Launched & $\%$ Change $^{b}$ \\
\hline Entries & 4195 & 466 & 700 & 229 & 884 & \\
\hline$C \log P$ & 3.23 & 3.00 & 3.24 & 2.57 & 2.50 & -23 \\
\hline$\% C \log P>5$ & 22.1 & 19.3 & 20.9 & 16.6 & 13.7 & -38 \\
\hline$\%$ Rule-of-five violations & 19.7 & 14.4 & 17.6 & 15.7 & 10.4 & -47 \\
\hline $\operatorname{PSA}\left(\AA^{2}\right)$ & 137 & 139 & 129 & 130 & 122 & -11 \\
\hline$\%$ PSA > $200 \AA^{2}$ & 24.1 & 22.7 & 20.0 & 22.3 & 18.6 & -23 \\
\hline MW (AMU) & 393 & 387 & 382 & 361 & 338 & -14 \\
\hline$\% M W>500$ & 22.9 & 17.8 & 19.1 & 14.8 & 11.2 & -51 \\
\hline No. Rotatable bonds & 7 & 7 & 6 & 6 & 6 & -14 \\
\hline$\%$ Rotatable bonds > 10 & 24.2 & 23.4 & 21.0 & 20.5 & 16.2 & -33 \\
\hline Andrews' BE (kcal/mol) & 12.5 & 11.5 & 12.8 & 10.6 & 10.5 & -16 \\
\hline No. H-bond acceptors & 4 & 4 & 4 & 4 & 4 & 0 \\
\hline No. H-bond donors & 2 & 2 & 2 & 2 & 1 & -50 \\
\hline
\end{tabular}

in Table 2 are significant when comparing the Preclinical and Launched categories. For example, the median Clog $P$ of Preclinical compounds is 3.23 , while the corresponding value for launched compounds is $23 \%$ lower or 2.50 . The most significant changes seem to occur for the out-of-range properties. In particular, the success of compounds entering clinical development with a $C \log P>5, \mathrm{MW}>500$, rotatable bonds $>10$, or rule-of-five violations seem to be heavily disfavored (illustrated in Figure 1). The most significant change is observed for compounds with $\mathrm{MW}>500$, where their representation in the

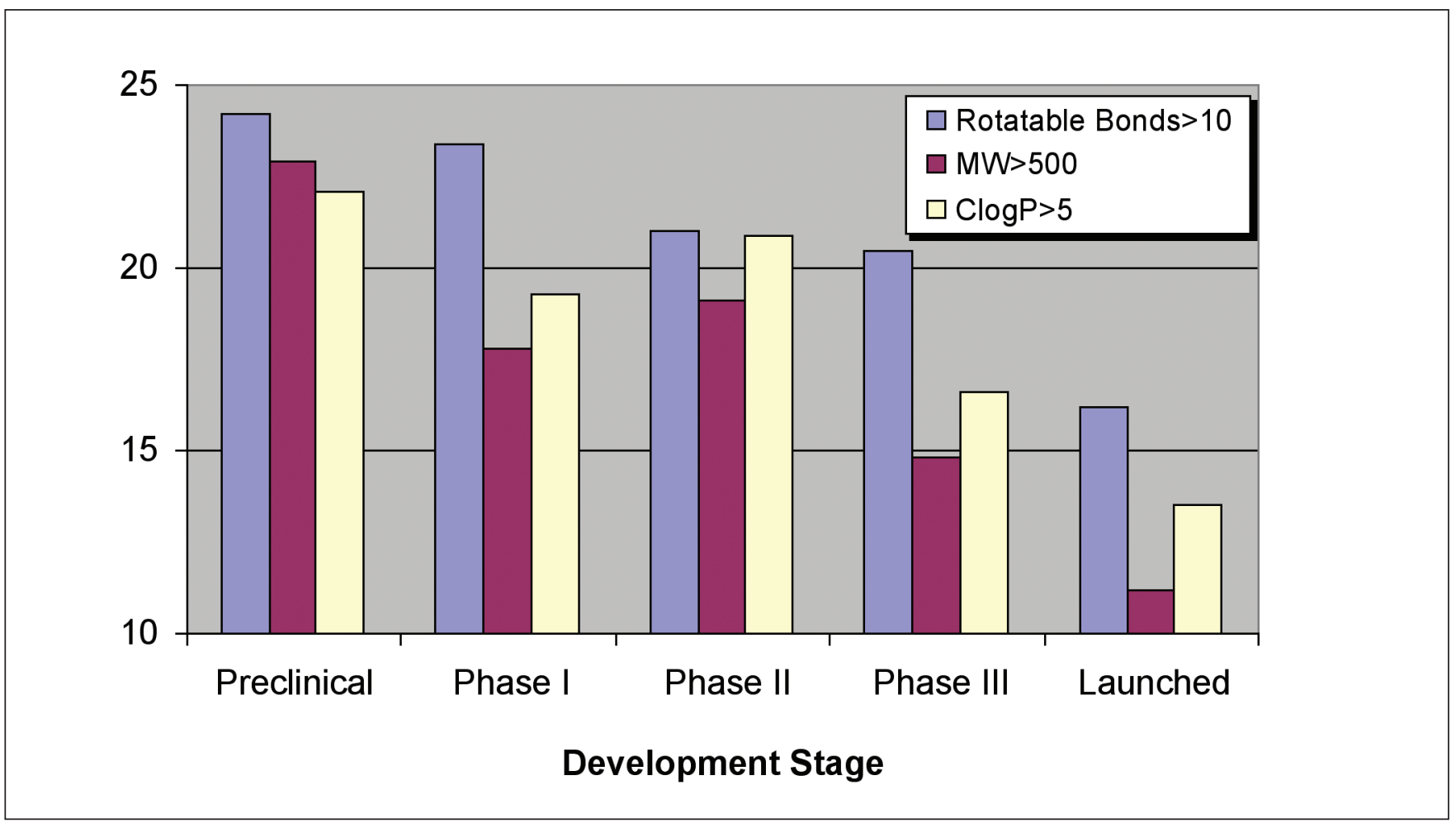

Figure 1. Histogram of the percentage of compounds that lie outside the given cut off values vs. the stage of clinical development. 
Launched category is 51\% lower than Preclinical.

How much does the rule-of-five account for? Clearly, compounds that are not rule-of-five compliant have low chances of survival ( $47 \%$ decrease in representation from Preclinical to Launched status). In the present study, a total of 1144 compounds (826 of these are in the Preclinical category) are not rule-of-five compliant (two or more violations of any parameters) (6). However, if all of these compounds were eliminated from the study, the same selection pattern toward better computed physical properties would still be observed. For example, the median $\operatorname{Cog} P$ for Preclinical, Phase I, Phase II, Phase III, and Launched compounds would be 3.06, 2.88, 3.00, 2.40, and 2.50 , respectively. In this case, an $18 \%$ decrease is computed from Preclinical to Launched status for the rule-of-five compliant subset of compounds (vs. $23 \%$ reported in Table 2). Similar trends are also observed for the other properties of interest-in each case there is a precipitous drop in the median property value in advancing from Preclinical to Launched status with most of the change occurring in the latter stages of development (excessive MW is the one exception for this subset, since most of these compounds were eliminated from consideration by the rule-of-five filtering).

These results suggest that certain computed physical properties are selected for during clinical development of human therapeutic agents. What is most striking is that the selection process tends to occur in the latter stages of development (Phase II and beyond), when failures are more costly both in terms of time and resources. This is particularly interesting, since one would assume that by the time a Preclinical or Phase I investigation is started, there has already been significant effort expended toward choosing the best compound for development. From the present analysis, significant risks are associated with selecting compounds for development that lie outside the cutoff values in Table 2 .

Obviously, compounds will fail during clinical development for any number of reasons, such as failure to show anticipated biological effects, toxicity, etc. (29). Good properties are but one element of a quality compound or lead. Since on average only four new-in-class drugs are launched each year (30), despite increasingly intense industry-wide attention to absorption, distribution, metabolism, and excretion (ADME)-related properties in discovery, it is clear that choosing the best lead to follow-up is critical to future success.

\section{IMPLICATIONS FOR COMBINATORIAL LIBRARY DESIGN AND LEAD SELECTION}

How do the current results affect our thinking toward suitable properties for combinatorial library design and the selection of leads for further optimization? In many respects, the current study reinforces the notion that compounds with certain physical properties have a greater chance of succeeding in the clinic and becoming drugs. Thus, synthesizing compounds with physical properties distributed around the ranges given by the Phase III and Launched values in Table 2 should increase the chances of finding leads with enhanced survivability characteristics. Perhaps more important is ensuring that excessive numbers of compounds $(>15 \%)$ do not lie outside the property cutoff ranges above, as these compounds do not fare well in terms of success in the clinic.

\section{LIBRARY DESIGN EXAMPLE}

Consider the construction of a combinatorial library based on a 2,5-diketopiperazine motif (31).

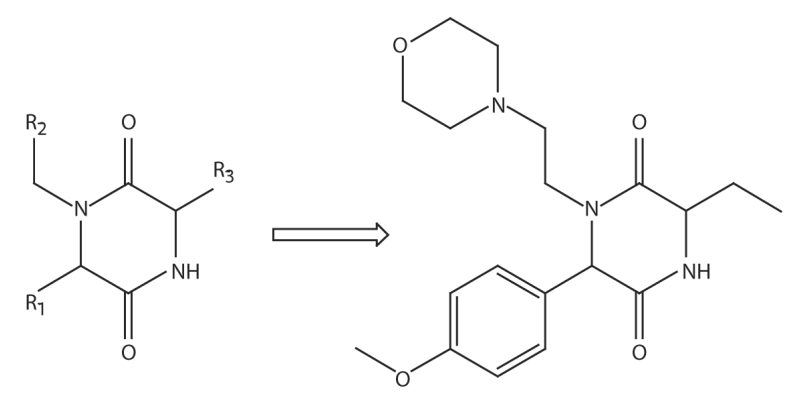

Based on the chemistry used to construct the library, a wide range of substituents may be placed at $\mathrm{R}_{1}$ (aldehydes), $\mathrm{R}_{2}$ (primary amines), and $\mathrm{R}_{3}$ (boc-protected amino acids). In the $\mathrm{ab}-$ sence of any specific structural information that could be used to guide the selection of the components, we base our choices on a combination of diversity and physical property considerations. In the three-component example above, we can construct a 4000 compound diversity library with the following properties (median values listed): (i) MW 357 (0.36\% with MW > 500); 
(ii) $\operatorname{Clog} P 3.38$ (3\% with $C \log P>5$ ); (iii) PSA $80.5 \AA^{2}(2.5 \%$ with PSA > $200 \AA^{2}$ ); and (iv) number of rotatable bonds is 6 , with $100 \%$ rule-of-five compliance.

In designing combinatorial libraries, the chemist is often faced with a large number of possible choices and the ability to manipulate many variables. A thoughtful strategy should include close attention to the property distributions of the final products, as this will have the greatest impact on the future success of any lead optimization program.

\section{REFERENCES}

1.Blake, J.F. 2002. Structure-based generation of viable leads from small combinatorial libraries. Advances in Structure-Guided Drug Discovery. La Jolla, CA, November 13-15.

2.Golebiowski, A., S.R. Klopfenstein, and D.E. Portlock. 2001. Lead compounds discovered from libraries. Curr. Opin. Chem. Biol. 5:273-284.

3.Spencer, R.W. 1998. High-throughput screening of historic collections: observations on file size, biological targets, and file diversity. Biotech. Bioeng. 61:61-67.

4.Lipinski, C.A. 2000. Drug-like properties and the causes of poor solubility and poor permeability. J. Pharmacol. Toxicol. Methods 44:235-249.

5.Lipinski, C.A. 1999. Consideration of drug solubility in high throughput screening of oral drug candidates, in Strategies for Optimizing Oral Drug Delivery: Scientific to Regulatory Approaches. April 19-21, Kobe, Japan.

6.Lipinski, C.A., F. Lombardo, B.W. Dominy, and P.J. Feeney. 1997. Experimental and computational approaches to estimate solubility and permeability in drug discovery and development settings. Adv. Drug Delivery Rev. 23:3-25.

7.Ajay, A., W.P. Walters, and M.A. Murcko. 1998. Can we learn to distinguish between "drug-like" and "nondrug-like" molecules? J. Med. Chem. 41:33143324 .

8.Walters, W.P. and M.A. Murcko. 2002. Prediction of 'drug-likeness'. Adv. Drug Deliv. Rev. 45:255-271.

9.Sadowski, J. and H.A. Kubinyi. 1998. Scoring scheme for discriminating between drugs and nondrugs. J. Med. Chem. 41:3325-3329.

10.Oprea, T.I. 2000. Property distribution of drug-related chemical databases. J. Comput. Aided Mol. Des. 14:251-264.

11.Muegge, I., S.L. Heald, and D. Britelli. 2001. Simple selection criteria for drug-like chemical matter. J. Med. Chem. 44:1841-1846.

12.Brüstle, M., B. Beck, T. Schindler, W. King, T. Mitchell, and T. Clark. 2002. Descriptors, physical properties, and drug-likeness. J. Med. Chem. 45:3345-3355.

13.Tounge, B.A., L.B. Pfahler, and C.H. Reynolds. 2002. Chemical information based scaling of molecular descriptors: a universal chemical scale for library design and analysis. J. Chem. Inf. Comput. Sci. 42:879-884.

14.Wessel, M.D., P.C. Jurs, J.W. Tolan, and S.M. Muskal. 1998. Prediction of human intestinal absorption of drug compounds from molecular structure. J. Chem. Inf. Comput. Sci. 38:726-735.

15.Yoshida, F. and J.G. Topliss. 2000. QSAR model for drug human bioavailability. J. Med. Chem. 43:2575-2585.

16.Veber, D.F., S.R. Johnson, H.-Y. Cheng, B.R. Smith, K.W. Ward, and K.D. Kopple. 2002. Molecular properties that influence the oral bioavailability of drug candidates. J. Med. Chem. 45:2615-2623.

17.Oprea, T.I., A.M. Davis, S.J. Teague, and P.D. Leeson. 2001. Is there a difference between leads and drugs? A historical perspective. J. Chem. Inf. Comput. Sci. 41:1308-1315.

18.Hann, M.M., A.R. Leach, and G. Harper. 2001. Molecular complexity and its impact on the probability of finding leads for drug discovery. J. Chem. Inf. Comput. Sci. 41:856-864.

19.Proudfoot, J.R. 2002. Drugs, leads, and drug-likeness: an analysis of some recently launched drugs. Bioorg. Med. Chem. Lett. 12:1647-1650.

20.MDL Drug Data Report. 1999. MDL Information Systems, Inc., San Leandro, CA.

21.Blake, J.F. 2000. Chemoinformatics-predicting the physicochemical properties of 'drug-like' molecules. Curr. Opin. Biotechnol. 11:104-107.

22.Clark, D.E. and S.D. Pickett. 2000. Computational methods for the prediction of drug-likeness. Drug Discovery Today 5:49-58.
23. Wessel, M.D. and S. Mente. 2001. ADME by computer. Ann. Rev. Med. Chem. 36:257-266.

24.Andrews, PR, D.J. Craik, and J.L. Martin. 1984. Functional group contributions to drug-receptor interactions. J. Med. Chem. 27:1648-1657.

25.Skell, J.M. and R.S. Pearlman. SAVOL2, July 1988, University of Texas at Austin, Austin, TX. Van der Waals parameters were obtained by scaling the OPLS-AA sigma values by $2^{(1 / 6) / 2}$. OPLS-AA parameters from Jorgensen, W.L., D.S. Maxwell, and J. Tirado-Rives. 1996. Development and testing of the OPLS all-atom force field on conformational energetics and properties of organic liquids. J. Am. Chem. Soc. 118:11225-11236.

26.R, version 1.6.1. Freely available from (http://www.r-project.org).

27.Royston, P. 1982. An extension of Shapiro and Wilk's W test for normality to large samples. Appl. Stat. 31:115-124.

28.Bauer, D.F. 1972. Constructing confidence sets using rank statistics. J. Amer. Stat. Assoc. 67:687-690.

29.Venkatesh, S. and R.A. Lipper. 2000. Role of the development scientist in compound lead selection and optimization. J. Pharm. Sci. 89:145-154.

30.Hopkins, A.L. and C.R. Groom. 2002. The druggable genome. Nat. Rev. Drug Discov. 1:727-730.

31.Kennedy, A.L., A.M. Freyer, and J.A. Josey. 2002. A new resin-bound universal isonitrile for the Ugi 4CC reaction: preparation and applications to the synthesis of 2,5-diketopiperazines and 1,4-benzodiazepine-2,5-diones. Org. Lett. 4:1167-1170.

\section{Address correspondence to:}

James F. Blake

Array BioPharma, Inc.

3200 Walnut Street

Boulder, CO 80301, USA

e-mail:Jim.Blake@arraybiopharma.com 